Artigo de Revisão

\title{
岳
}

\section{Impacto dos conectores sem agulhas na infecção da corrente sanguínea: revisão sistemática*}

\author{
Impacto de los conectores sin agujas en la infección de la corriente sanguinea: revisión sistemática \\ Impact of needleless connectors in the bloodstream infection: a systematic review
}

\author{
Silvia Helena Frota Mendonça ${ }^{1}$, Rúbia Aparecida Lacerda ${ }^{2}$
}

\section{RESUMO}

Esta revisão sistemática buscou evidenciar o impacto do uso de conectores sem agulhas para sistema fechado de infusão na ocorrência de infecção da corrente sanguínea relacionada ao cateter venoso central. A amostra constitui-se de 14 estudos, os quais investigaram somente conectores sem agulhas. A infecção da corrente sanguínea relacionada ao cateter venoso central foi o desfecho de nove estudos. Seis apresentaram diferenças a favor do conector valvulado; quatro a favor do conector puncionável com cânula; um a favor do conector puncionável com agulha; um a favor do conector valvulado com pressão positiva e dois a favor do dispositivo usado antes da troca. A heterogeneidade dos estudos não permitiu a realização de metanálise.

Descritores: Literatura de revisão como assunto; Infecções relacionadas a cateter; Infecção hospitalar

\begin{abstract}
Esta revisión sistemática buscó evidenciar el impacto del uso de conectores sin agujas para sistemas cerrados de infusión en el caso de ocurrir una infección en la corriente sanguínea relacionada al catéter venoso central. La muestra fue constituida de 14 estudios, los cuales investigaron solamente conectores sin agujas. La infección de la corriente sanguínea relacionada al catéter venoso central fue el resultado de nueve estudios. Seis presentaron diferencias a favor del conector de válvula; cuatro a favor del conector para punción con cánula; uno a favor del conector para punción con aguja; uno a favor do conector de válvula con presión positiva y dos a favor del dispositivo usado antes del cambio. La heterogeneidad de los estudios no permitió la realización de una meta-análisis.
\end{abstract}

Keywords: Review literature as topic; Catheter-related infections; Cross infection

\section{RESUMEN}

This systematic review was intended to evaluate the impact of using needleless connectors in closed infusion systems in the event of a bloodstream infection related to central venous catheter. The sample consisted of 14 studies, which investigated only needleless connectors. The bloodstream infection related to central venous catheter was the result of nine studies. Six produced evidence in favor of the valve connector, four in favor of the cannula connector for puncturing, one in favor of needle connector, one in favor of positive pressure valve connector, and two in favor of the device used before the change. The heterogeneity of the studies did not allow the realization of a metaanalysis.

Descriptores: Literatura de revisión como asunto; Infecciones relacionadas con catéteres; Infección hospitalaria

\footnotetext{
* Extraída da Dissertação de Mestrado "Impacto do uso de conectores sem agulha para sistema fechado de infusão na ocorrência de infecção de corrente sanguinea relacionada ao cateter venoso central: evidências de uma revisão sistemática".

${ }^{1}$ Mestre em Enfermagem. Enfermeira Coordenadora dos Serviços Auxiliares de Diagnóstico e Terapêtica do Hospital Samaritano.

${ }^{2}$ Professora Associada da Escola de Enfermagem da Universidade de São Paulo - USP - São Paulo (SP), Brasil.
} 


\section{INTRODUÇÃO}

As infecções da corrente sanguínea relacionadas ao cateter venoso central (CVC) são em maior número, mais graves e ocorrem, principalmente, em pacientes de unidade de terapia intensiva (UTI). Pacientes em UTI têm múltiplos acessos ao CVC por dia, o que aumenta o risco de contaminação e subsequente infecção ${ }^{(1-2)}$.

O guideline para Prevenção de Infecção da Corrente Sanguínea relacionada ao cateter, elaborado pelo Centers for Disease Control (CDC), de 2002, recomenda a limitação das manipulações e das aberturas dos sistemas de infusão como medida de prevenção de infecção de corrente sanguínea ${ }^{(2)}$. Todavia, mesmo no sistema fechado a possibilidade da ocorrência de contaminação não é totalmente excluída, uma vez que são utilizados conectores, com ou sem agulhas, que atuam como intermediários entre o equipo e o cateter.

$\mathrm{Na}$ literatura não há evidências conclusivas sobre as vantagens do conector sem agulha para o paciente. Além disso, a definição de qual, dentre os produtos existentes, constitui o conector ideal para a manutenção do sistema fechado, e que possa garantir o menor impacto na assistência, não está claramente descrita.

A presente investigação teve como finalidade buscar evidências do impacto do uso de conectores de sistema fechado sem agulhas na ocorrência de infecção da corrente sanguínea relacionada ao cateter venoso central, por meio da revisão sistemática da literatura científica.

\section{MÉTODOS}

A revisão sistemática é um recurso importante da prática baseada em evidências, na qual os resultados das pesquisas são coletados, categorizados, avaliados e sintetizados ${ }^{(3)}$.

Para esta revisão foram incluídos os estudos com os componentes do PICO (P - População; I - Intervenção; $\mathbf{C}$ - controle; $\mathbf{O}$ - outcomes) preconizado por alguns autores $^{(4)}$ : População: estudos com pacientes em uso de cateter venoso central, independente de idade, sexo, etnia

Quadro 1. Escala de avaliação de qualidade dos estudos na área de Prevenção Controle e de Infecção da Corrente Sanguínea Relacionada ao cateter Venoso Central - EQIRC.

\begin{tabular}{|c|c|c|}
\hline $\begin{array}{l}\text { Grau de } \\
\text { recomendação }\end{array}$ & $\begin{array}{l}\text { Nível de } \\
\text { evidência }\end{array}$ & Estudos terapêuticos \\
\hline \multirow{6}{*}{ A } & $1 \mathrm{a}+$ & $\begin{array}{l}\text { Revisão sistemática (com homogeneidade) de ensaios clínicos controlados e randomizados, com } \\
\text { controle de todos os fatores intervenientes de risco proposto }\end{array}$ \\
\hline & $1 \mathrm{a}-$ & $\begin{array}{l}\text { Revisão sistemática (com homogeneidade) de ensaios clínicos controlados e randomizados, sem } \\
\text { controle de todos os fatores intervenientes de risco proposto }\end{array}$ \\
\hline & $1 b+$ & $\begin{array}{l}\text { Ensaios clínicos controlados e randomizados com intervalo de confiança estreito, com controle de } \\
\text { todos os fatores intervenientes de risco proposto }\end{array}$ \\
\hline & $1 b-$ & $\begin{array}{l}\text { Ensaios clínicos controlados e randomizados com intervalo de confiança estreito, sem controle de } \\
\text { todos os fatores intervenientes de risco proposto }\end{array}$ \\
\hline & $1 c+$ & $\begin{array}{l}\text { Resultados terapêuticos do tipo "Tudo ou Nada" com controle de todos os fatores intervenientes de } \\
\text { risco proposto }\end{array}$ \\
\hline & $1 c-$ & $\begin{array}{l}\text { Resultados terapêuticos do tipo "Tudo ou Nada" sem controle de todos os fatores intervenientes de } \\
\text { risco proposto }\end{array}$ \\
\hline \multirow{10}{*}{$\mathbf{B}$} & $2 a+$ & $\begin{array}{l}\text { Revisão sistemática (com homogeneidade) de estudos de coorte, com controle de todos os fatores } \\
\text { intervenientes de risco proposto }\end{array}$ \\
\hline & $2 a-$ & $\begin{array}{l}\text { Revisão sistemática (com homogeneidade) de estudos de coorte, sem controle de todos os fatores } \\
\text { intervenientes de risco proposto }\end{array}$ \\
\hline & $2 b+$ & $\begin{array}{l}\text { Estudo de coorte (incluindo ensaio clínico randomizado de menor qualidade), com controle de todos } \\
\text { os fatores intervenientes de risco proposto }\end{array}$ \\
\hline & $2 \mathrm{~b}-$ & $\begin{array}{l}\text { Estudo de coorte (incluindo ensaio clínico randomizado de menor qualidade), sem controle de todos os } \\
\text { fatores intervenientes de risco proposto }\end{array}$ \\
\hline & $2 \mathrm{c}+$ & $\begin{array}{l}\text { Estudo observacional de resultados terapêuticos (outcome research) e estudo ecológico, com controle de } \\
\text { todos os fatores intervenientes de risco proposto }\end{array}$ \\
\hline & $2 c-$ & $\begin{array}{l}\text { Estudo observacional de resultados terapêuticos (outcome research) e estudo ecológico, sem controle de } \\
\text { todos os fatores intervenientes de risco proposto }\end{array}$ \\
\hline & $3 a+$ & $\begin{array}{l}\text { Revisão sistemática (com homogeneidade) de estudos de caso-controle, com controle de todos os } \\
\text { fatores intervenientes de risco proposto }\end{array}$ \\
\hline & $3 a-$ & $\begin{array}{l}\text { Revisão sistemática (com homogeneidade) de estudos de caso-controle, sem controle de todos os } \\
\text { fatores intervenientes de risco proposto }\end{array}$ \\
\hline & $3 \mathrm{~b}+$ & Estudo de caso-controle, com controle de todos os fatores intervenientes de risco proposto \\
\hline & $3 \mathrm{~b}-$ & Estudo de caso-controle, sem controle de todos os fatores intervenientes de risco proposto \\
\hline C & 4 & $\begin{array}{l}\text { Relato de caso (incluindo coorte ou caso-controle de menos qualidade), com controle de todos os } \\
\text { fatores intervenientes de risco proposto }\end{array}$ \\
\hline D & 5 & $\begin{array}{l}\text { Opinião desprovida de avaliação crítica, baseada em consensos, estudos fisiológicos, com materiais } \\
\text { biológicos ou modelos animais }\end{array}$ \\
\hline
\end{tabular}

Adaptado de Nobre M, Bernardo W. Prática clínica baseada em evidência. Rio de Janeiro: Elsevier; $2006{ }^{(5) .}$ 
e serviço de saúde vinculado; Intervenção: uso de conectores de sistema fechado sem agulhas, não-valvulados, valvulados, com pressão positiva ou não; Comparação: uso de oclusores, conectores de sistema fechado com ou sem agulhas, nãovalvulados, valvulados, com pressão positiva ou não; Outcome (desfecho): infecção da corrente sanguínea, contaminação do canhão, contaminação microbiana, infecção de corrente sanguínea relacionada ao cateter,

As fontes de busca dos estudos foram: bases de dados eletrônicas (PubMed/MEDLINE, OVID, EMBASE, LILACS, CINAHL, Cochrane) e as referências bibliográficas dos estudos incluídos. A qualidade dos estudos foi analisada quanto a sua força da evidência, utilizando duas escalas de avaliação: Escala de Estudos da Área de Controle e Prevenção de Infecção da Corrente Sanguínea Relacionada ao cateter Venoso Central (EQIRC), de acordo com o desenho de pesquisa e no controle de fatores intervenientes, proposto pelas autoras baseadas na escala adaptada de Nobre e Bernardo ${ }^{(5)}$; Escala de Jadad ${ }^{(6)}$. O Quadro 1 apresenta a EQIRC.

Para determinar o grau de recomendação dos estudos, utilizou-se a classificação proposta por Oxford for Center Evidence-Based Medicine ${ }^{(7)}$. Os graus de recomendação são classificados como A - evidência forte; $\mathbf{B}$ - evidência moderada; $\mathbf{C}$ - evidência fraca; $\mathbf{D}$ - evidência muito fraca.

\section{RESULTADOS}

A busca nas bases de dados forneceu, na fase de pré- seleção, 266 estudos e após reunião de consenso, 14 estudos foram incluídos na revisão sistemática, os quais serão designados pela letra " $E$ " seguidos pelo número atribuído ao estudo, conforme a busca realizada para esta investigação.

As variáveis independentes mais investigadas foram: uso somente de conectores e uso de conectores e acessórios (extensão e/ ou cânulas) juntamente com a substância anti-séptica usada para desinfecção $(28,6 \%)$. Com relação ao tipo de conector e acessório pesquisados, houve grande variação entre os estudos (11 combinações para 14 estudos).

Dos fatores intrínsecos, idade $(78,6 \%)$ e sexo $(71,4 \%)$ obtiveram maiores percentuais. Os estudos que mais controlaram fatores intrínsecos foram os E75 (10), E117 (9), E73 (6) e E69 (5). Destaca-se que, com exceção dos E75 e E117, os demais não caracterizavam ensaios clínicos randomizados, considerados como os de melhor categoria de evidência.

Quanto ao controle de fatores extrínsecos, devido à sua variedade, eles foram previamente classificados em categorias. Com relação à categoria do conector, foram identificados nove tipos de fatores controlados, sendo que os mais utilizados corresponderam à freqüência de troca dos dispositivos $(85,7 \%)$, tipo de conector $(78,6 \%)$, substância para desinfecção do conector $(71,4 \%)$, pelos seguintes estudos: E65, E69 e E74 (5 cada) e E42, E70, E75, E89 e E117 (4 cada).

$\mathrm{Na}$ categoria relativa ao cateter venoso central, os fatores extrínsecos mais controlados foram: tipo de cateter

Quadro 2. Síntese Panorâmica dos principais dados analisados nos Estudos incluídos na Revisão Sistemática. São Paulo, 2007.

\begin{tabular}{|c|c|c|c|c|c|c|c|c|}
\hline Est. & $\begin{array}{c}\text { Tipo } \\
\text { investigação }\end{array}$ & Intervenção & Controle & EQIRC & Jadad & $\begin{array}{c}\text { Variáveis } \\
\text { controladas }\end{array}$ & $\begin{array}{l}\text { Diferença na } \\
\text { colonização e } \\
\text { contaminação }\end{array}$ & $\begin{array}{l}\text { Diferença de } \\
\text { ICS-RC }\end{array}$ \\
\hline E79 & $\begin{array}{l}\text { Ensaio clínico } \\
\text { controlado } \\
\text { randomizado }\end{array}$ & $\begin{array}{l}\text { Conector com } \\
\text { injetor pré- } \\
\text { furado com } \\
\text { cânula }\end{array}$ & $\begin{array}{l}\text { Dânula/ } \\
\text { extensão } \\
\text { com } \\
\text { Oclusor }\end{array}$ & $2 \mathrm{~b}-$ & 03 & 19 & $\begin{array}{c}\text { Sem SE } \text { SEara }^{2} \text { pasiva de } \\
\text { cultura positiva do } \\
\text { ponta e canhão do } \\
\text { cateter }\end{array}$ & $\begin{array}{c}\text { Sim, a favor da } \\
\text { intervenção } \\
\text { (IC95\%: } 0,86- \\
55,87)\end{array}$ \\
\hline E21 & $\begin{array}{l}\text { Ensaio clínico } \\
\text { controlado } \\
\text { randomizado }\end{array}$ & $\begin{array}{l}\text { Conector } \\
\text { valvulado }\end{array}$ & Oclusor & $1 b-$ & 01 & 17 & $\begin{array}{c}\text { Sim, a favor da } \\
\text { intervenção } \\
\text { IC }(0,70-2,61) \\
p=0,36\end{array}$ & $\begin{array}{c}\text { Sim, a favor da } \\
\text { intervenção } \\
\text { IC }(0,63-2,69) \\
p=0,5\end{array}$ \\
\hline E75 & $\begin{array}{l}\text { Ensaio clínico } \\
\text { controlado } \\
\text { randomizado }\end{array}$ & $\begin{array}{l}\text { Conector } \\
\text { valvulado }\end{array}$ & Oclusor & $2 \mathrm{~b}-$ & 01 & 29 & $\begin{array}{c}\text { Sim, a favor da } \\
\text { intervenção. } \\
(6,5 \% \times 9,3 \% ; \mathrm{p}= \\
0,37)\end{array}$ & $\begin{array}{c}\text { Sim, a favor da } \\
\text { intervenção } \\
(\text { IC95\%: } 0,88- \\
58,0) p=0,03\end{array}$ \\
\hline E117 & $\begin{array}{l}\text { Ensaio clínico } \\
\text { controlado } \\
\text { randomizado }\end{array}$ & $\begin{array}{l}\text { Conector } \\
\text { valvulado }\end{array}$ & Oclusor & $1 b-$ & 04 & 36 & $\begin{array}{c}\text { Sim, a favor da } \\
\text { intervenção, IC } \\
95 \%(0,12-0,63) \\
\text { p }<0,0017\end{array}$ & $\begin{array}{c}\text { Sim, a favor da } \\
\text { intervenção } \\
(3,4 \% \times 6,3 \%, \\
\text { p=0,22) }\end{array}$ \\
\hline E65 & $\begin{array}{l}\text { Ensaio clínico } \\
\text { controlado } \\
\text { randomizado }\end{array}$ & $\begin{array}{c}\text { Conector } \\
\text { valvulado com } \\
\text { extensão } \\
\end{array}$ & $\begin{array}{l}\text { Oclusor } \\
\text { com dânula }\end{array}$ & $1 b-$ & 03 & 13 & $\begin{array}{c}\text { Sim, a favor da } \\
\text { intervenção } \\
p<0,0001 \\
\end{array}$ & \\
\hline E89 & $\begin{array}{l}\text { Ensaio clínico } \\
\text { controlado } \\
\text { randomizado }\end{array}$ & $\begin{array}{c}\mathrm{CV}^{3} \text { com } \\
\text { pressão positiva } \\
\text { e } 3 \text { antissép- } \\
\text { ticos diferentes }\end{array}$ & $\begin{array}{l}\text { Oclusor e } 3 \\
\text { antissép- } \\
\text { ticos } \\
\text { diferentes }\end{array}$ & $2 \mathrm{~b}-$ & 01 & 11 & $\begin{array}{c}\text { Sim, a favor da } \\
\text { intervenção. Sem } \\
\text { IC. }\end{array}$ & \\
\hline
\end{tabular}




\begin{tabular}{|c|c|c|c|c|c|c|c|c|}
\hline Est. & $\begin{array}{c}\text { Tipo } \\
\text { investigação }\end{array}$ & Intervenção & Controle & EQIRC & Jadad & $\begin{array}{l}\text { Variáveis } \\
\text { controladas }\end{array}$ & $\begin{array}{l}\text { Diferença na } \\
\text { colonização e } \\
\text { contaminação }\end{array}$ & $\begin{array}{l}\text { Diferença de } \\
\text { ICS-RC }\end{array}$ \\
\hline E42 & Coorte & $\begin{array}{l}\text { Conector } \\
\text { valvulado } \\
\text { com pressão } \\
\text { positiva }\end{array}$ & $\begin{array}{l}\text { Conector } \\
\text { com } \\
\text { injetor } \\
\text { pré-furado } \\
\text { com } \\
\text { cânula } \\
\end{array}$ & $2 b-$ & $\begin{array}{l}\text { Não } \\
\text { se } \\
\text { aplica }\end{array}$ & 15 & & $\begin{array}{c}\text { Sim, a favor do } \\
\text { controle }(8,8 \text { X } \\
15,5 \text { ICS-RC / } \\
1000 \text { cateter-dia, } \\
\text { com SE, não } \\
\text { apresenta IC95\%) }\end{array}$ \\
\hline E72 & Coorte & $\begin{array}{l}\text { Conector } \\
\text { valvulado }\end{array}$ & $\begin{array}{l}\text { Sem } \\
\text { especifi- } \\
\text { cação }\end{array}$ & $2 \mathrm{~b}-$ & $\begin{array}{l}\text { Não } \\
\text { se } \\
\text { aplica }\end{array}$ & 04 & & 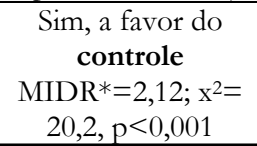 \\
\hline E74 & Coorte & $\begin{array}{l}\text { Conector com } \\
\text { injetor pré- } \\
\text { furado com } \\
\text { cânula }\end{array}$ & $\begin{array}{l}\text { Conector } \\
\text { PRN }{ }^{\circledR} \\
\text { com } \\
\text { agulha }\end{array}$ & $2 \mathrm{~b}-$ & $\begin{array}{l}\text { Não } \\
\text { se } \\
\text { aplica }\end{array}$ & 14 & & $\begin{array}{c}\text { Sim, a favor do } \\
\text { controle e maior } \\
\text { para o uso do } \\
\text { dispositivo } \\
\text { intervenção e } \\
\text { NPT juntos }(3 / 87 \\
\text { X } 8 / 15 ; \mathrm{RR}=9,45 ; \\
\text { IC } 95 \% 4,3-21,0), \\
\mathrm{p}<0,001 \\
\end{array}$ \\
\hline E73 & Coorte & $\begin{array}{l}\text { Conector } \\
\text { valvulado }\end{array}$ & $\begin{array}{l}\text { Sem } \\
\text { especifi- } \\
\text { cação }\end{array}$ & $2 \mathrm{~b}-$ & $\begin{array}{l}\text { Não } \\
\text { se } \\
\text { aplica }\end{array}$ & 19 & & $\begin{array}{c}\text { Sim, a favor do } \\
\text { controle, }(0,8 \times 1,4 \\
\text { ICS-RC / } 1000 \\
\text { cateter-dia, } \\
\text { RR=1,8, IC 95\% } \\
1,12-2,89), \\
\text { p }<0,02 \\
\end{array}$ \\
\hline E76 & $\begin{array}{l}\text { Resultado } \\
\text { Terapêutico }\end{array}$ & $\begin{array}{c}\text { Conector } \\
\text { valvulado } \\
\text { com pressão } \\
+\end{array}$ & $\begin{array}{l}\text { Conector } \\
\text { valvulado }\end{array}$ & $2 c-$ & $\begin{array}{l}\text { Não } \\
\text { se } \\
\text { aplica }\end{array}$ & 03 & & $\begin{array}{c}\text { Sim, a favor do } \\
\text { controle } \\
(1,5 \times 2,4 \text { ICS-RC / } \\
1000 \text { cateter-dia; IC } \\
\mathbf{9 5 \%} 1,04-2,48), \\
\text { p=0,03 }\end{array}$ \\
\hline E69 & $\begin{array}{l}\text { Caso- } \\
\text { controle }\end{array}$ & $\begin{array}{c}2 \text { Conectores } \\
\text { valvulados } \\
(\mathrm{CV})\end{array}$ & $\begin{array}{l}\text { Conector } \\
\text { com } \\
\text { injetor } \\
\text { pré-furado }\end{array}$ & $3 b-$ & $\begin{array}{l}\text { Não } \\
\text { se } \\
\text { aplica }\end{array}$ & 17 & & $\begin{array}{c}\text { Sim, a favor do } \\
\text { controle para os } 2 \\
\text { CV, porém apenas } \\
1 \text { com } \mathbf{S E}(0,3 \mathrm{X} \\
1,4 \mathrm{ICS}-\mathrm{RC} / 1000 \\
\text { cateter-dia, OR }=4,5 \\
\text { (IC } 95 \% 1,9- \\
10,5), \mathrm{p}<0,01 \\
\end{array}$ \\
\hline E70 & $\begin{array}{c}\text { Caso- } \\
\text { controle }\end{array}$ & $\begin{array}{l}\text { Conector } \\
\text { valvulado } \\
\text { c/oclusor }\end{array}$ & $\begin{array}{l}\text { Conector } \\
\text { com } \\
\text { injetor } \\
\text { pré-furado }\end{array}$ & $3 b-$ & $\begin{array}{l}\text { Não } \\
\text { se } \\
\text { aplica }\end{array}$ & 23 & & $\begin{array}{l}\text { Sim, a favor do } \\
\text { controle } \\
\text { (IC 95\% 1,02 - } \\
2,54), \mathrm{p}=0,05\end{array}$ \\
\hline E101 & $\begin{array}{c}\text { Caso- } \\
\text { controle }\end{array}$ & $\begin{array}{l}\text { Conector } \\
\text { valvulado } \\
\text { com dânulas }\end{array}$ & Oclusor & $3 b-$ & $\begin{array}{l}\text { Não } \\
\text { se } \\
\text { aplica }\end{array}$ & 05 & $\begin{array}{l}\text { Sim, a favor do } \\
\text { intervenção para } \\
\text { contaminação } \\
\text { externa após } \\
\text { desinfecção com } \\
\text { álcool ( }<<0,0001) \\
\end{array}$ & \\
\hline
\end{tabular}

${ }^{1}$ IC - Intervalo de confiança ; ${ }^{2}$ SE - Significância estatística; ${ }^{3} \mathbf{C V}$ - Conector valvulado

* MIDR - Mantel-Haenszel Incidence Density Rate

$(57,1 \%)$, duração da cateterização $(50,0 \%)$, uso de Nutrição Parenteral Total, lipídios, quimioterapia e sangue (50,0\%), uso de antibioticoterapia endovenosa (42,9\%). Os estudos que mais controlaram esses fatores extrínsecos foram os E42 (7), E75 (6), E21 e E73 (5 cada) e E117(4).

$\mathrm{Na}$ categoria relativa à inserção/remoção do cateter, dos onze fatores identificados entre os estudos, destacaram-se: sítio de inserção $(50,0 \%)$ e substância para preparo da pele, razão da remoção do cateter e local de inserção do cateter $(28,6 \%)$. Na categoria cuidados com o cateter venoso central, dentre os cinco fatores identificados, a freqüência de troca do curativo foi o mais controlado (42,9\%), pelo estudo E70 (3).

O Quadro 2 distribui os principais estudos segundo o 
tipo de conector investigado, delineamento de estudo, resultado das avaliação aplicadas, tipo de desfecho e resultados dos estudos.

\section{DISCUSSÃO}

A incorporação de uma nova tecnologia em saúde implica em avaliar todas as nuances de interferência na práxis assistencial atual. Os estudos com delineamento adequado e resultados estatisticamente comprovados devem prover as evidências científicas que fundamentam a tomada de decisão.

Todas as pesquisas incluídas foram desenvolvidas in vivo. O papel da contaminação do canhão do cateter, como um fator que antecede e favorece a ocorrência de ICS-RC, está bem estabelecido ${ }^{(8-9)}$, no entanto, os estudos in vitro, apesar de utilizarem solução desafio, desprezavam a possibilidade dessa contaminação, durante a manipulação do dispositivo ${ }^{(10)}$.

A fase de produção dos estudos incluídos ocorreu a partir da década de 1990, o que coincide com o alerta emitido pelo Food and Drug Administration, em 1992, sobre os riscos de contaminação dos profissionais de saúde com o vírus da imunodeficiência humana - HIV, o vírus da hepatite $\mathrm{B}-\mathrm{HBV}$ e o vírus da hepatite $\mathrm{C}-\mathrm{HCV}$ por acidente perfuro-cortante com agulhas ${ }^{(11)}$.

Parcela importante $(50,0 \%)$ dos estudos (E42, E69, E70, E72, E73, E74, E76) analisaram apenas o desenvolvimento da infecção da corrente sanguínea relacionada a cateter. Três $(21,4 \%)$ analisaram apenas a contaminação do conector e do canhão do cateter (E65, $\mathrm{E} 89, \mathrm{E} 101)$. Somente quatro estudos $(28,6 \%)$ verificaram ambos os desfechos, infecção da corrente sanguínea e colonização, seja da ponta e do canhão do cateter, seja da pele (E21, E75, E79, E117).

Embora os critérios para análise dos desfechos tenham variado bastante entre os estudos, cada um deles utilizou os mesmos em todas as suas populações, ou seja, houve padronização dos critérios para a obtenção dos resultados, critérios esses aceitáveis, do ponto de vista da questão desta revisão sistemática.

As populações dos estudos desta revisão constituíram amostras não-aleatórias, o que corrobora a validade interna dos estudos ${ }^{(12)}$.

Outro aspecto que pode interferir sobremaneira na validade interna de estudos dessa natureza é o controle de todas ou as principais variáveis externas, realmente capazes de interferir no desfecho, além da intervenção. $\mathrm{Na}$ área de controle da infecção hospitalar, as variáveis externas correspondem, principalmente, aos diversos fatores de risco de infecção ${ }^{(2,13)}$. A quantidade de fatores extrínsecos controlados não implica necessariamente que os estudos de melhores evidências tenham sido incluídos. É importante ressaltar que os estudos controlaram outros fatores intervenientes, além daqueles considerados de principais evidências pelo CDC. Muitas dessas variáveis se relacionavam às características de populações específicas, selecionadas pelos estudos. Os E117 e E75 estão entre os que mais as controlaram.

Pela Escala EQIRC, a melhor classificação obtida foi o nível de evidência 1b- (E21, E65, E117). Tal nível situase no grau de recomendação A (evidência forte), ou seja, estudos experimentais de maior consistência.

É importante enfatizar que os estudos E21, E65 e E117 apresentaram o melhor tipo de desenho, porém, não controlaram todos os fatores de risco previamente considerados pelas recomendações do CDC para prevenção de infecção da corrente sangüínea relacionada a cateter venoso central.

Os E117 e o E21, ambos tendo obtido o mesmo nível de classificação (1b-), apresentaram discrepância com relação ao controle dos fatores de risco. O primeiro controlou um maior número de fatores dentre todos os estudos (36), sendo somente seis recomendados pelo CDC. O segundo controlou 17 fatores, dentre os quais, oito constituíram fatores com base nas recomendações do CDC. O estudo E89 obteve classificação 2b-, pois apesar de se tratar de um ensaio clínico controlado randomizado, não controlou todos os fatores intervenientes e não apresentou intervalo de confiança do resultado obtido, permanecendo, assim, na mesma classificação dos estudos de coortes.

Os estudos dessa revisão sistemática com melhores resultados na Escala de Jadad ${ }^{(6)}$ foram o E117 (4 pontos) e os E65 e E79 (3 pontos cada). Os demais obtiveram apenas 1 ponto (E21, E75, E89).

Observa-se coincidência entre as duas escalas utilizadas, de melhor estudo, somente para o E117. O E65, também recebeu 1b- na EQIRC, mas obteve pontuação 3 na Escala de $\operatorname{Jadad}^{(6)}$. O E79, classificado como 2b- na EQIRC, pois apresentou intervalo de confiança largo para o resultado obtido (IC 95\% - 0,89-55,87), também recebeu a pontuação 3 na Escala de $\operatorname{Jadad}^{(())}$. No E75 ocorreu situação oposta; sua classificação na primeira escala foi 2b-, entretanto foi avaliado como estudo pobre na escala de $\operatorname{Jadad}^{(()}$.

Considerando os aspectos avaliados, inclusive nas escalas aplicadas, dentre os estudos melhores classificados, os E65 e E117 (evidência forte) apresentaram diferença no índice de contaminação do conector e do canhão do cateter a favor dos conectores sem agulha valvulados (sistema fechado de infusão) quando comparados aos oclusores (sistema aberto de infusão). O E117 também demonstrou que o dispositivo da intervenção constituiu fator de proteção independente para colonização do canhão do cateter. O E75 (evidência moderada) comparou também conector valvulado e oclusor e obteve resultado favorável ao conector com manutenção do sistema fechado, no que diz respeito à diferença de índice de infecção da corrente sanguínea relacionada a cateter venoso central. 
O E79 (evidência moderada) comparou conector com injetor pré-furado com cânula (sistema fechado) e oclusor (sistema aberto), com resultado a favor do dispositivo de intervenção e também resultou como fator de proteção contra colonização do canhão do cateter.

$\mathrm{Na}$ presente revisão os melhores estudos encontrados constituem ensaios clínicos randomizados (E65 e E117, E75, E79), porém, a partir dos aspectos analisados, é necessário ressaltar que as evidências não dependem apenas do tipo de delineamento da pesquisa. Estudo holandês ${ }^{(14)}$ de revisão sobre essa temática, encontrado na literatura, também reconheceu problemas de condução das pesquisas analisadas. Além disso, a busca de evidências sobre práticas assistenciais específicas, como na área de controle de infecção, exige, além da necessidade de atender aos critérios de elaboração dos ensaios clínicos controlados e randomizados, também o controle dos diversos fatores de risco de infecção, capazes de interferir nos resultados, além daquele sob intervenção ${ }^{(15)}$.

É possível afirmar que há lacuna científica representada pela quase inexistência de estudos que comparam os diversos tipos de conectores sem agulha para sistemas

\section{REFERÊNCIAS}

1. Fernandes AT, Ribeiro Filho N. Infecção do acesso vascular. In: Fernandes AT, Fernandes MOV, Ribeiro Filho N. Infecção hospitalar e suas interfaces na área da saúde. São Paulo: Atheneu; 2000. v.1. p. 556-79.

2. O'Grady NP, Alexander M, Dellinger EP, Gerberding JL, Heard SO, Maki DG, et al. Guidelines for the prevention of intravascular catheter-related infections. Centers for Disease Control and Prevention. MMWR Recomm Rep. 2002;51(RR10):1-29.

3. Sackett DL, Straus SE, Richardson WS, Rosenberg W, Haynes RB. Medicina baseada em evidências: prática e ensino. 2a. ed. Porto Alegre: Artmed; 2003.

4. Nobre MRC, Bernardo WM, Jatene FB. A prática clínica baseada em evidências. Parte I - questões clínicas bem construídas. Rev Assoc Med Bras (1992). 2003;49(4):445-9.

5. Nobre M, Bernardo W. Prática clínica baseada em evidência. Rio de Janeiro: Elsevier; 2006.

6. Jadad AR, Moore RA, Carroll D, Jenkinson C, Reynolds DJ, Gavaghan DJ, McQuay HJ. Assessing the quality of reports of randomized clinical trials: is blinding necessary? Control Clin Trials. 1996;17(1):1-12.

7. Center for Evidence Based Medicine - CEBM. Levels of Evidence and Grades of Recommendations [Internet]. [cited 2010 Mar 26]. Available from: <http://www.cebm.net>

8. Diener JRC, Coutinho MSSA, Zoccoli CM. Infecções relacionadas ao cateter venoso central em terapia intensiva. Rev Assoc Med Bras (1992). 1996;42(4):205-14.

9. Mermel LA. Prevention of intravascular catheter-related fechados entre si. Nesta revisão, o conector sem agulha valvulado com pressão positiva não foi avaliado por nenhum estudo mais consistente.

A ausência de homogeneidade entre pelo menos dois estudos incluídos nesta revisão, principalmente referente às intervenções e desfechos, não viabilizou a realização de metanálise.

\section{CONCLUSÃO}

Com base nos quatro melhores estudos encontrados, é possível afirmar que a implantação de conectores sem agulhas, quer valvulados ou puncionáveis, com manutenção de sistema fechado de infusão, apresentam impacto positivo na menor contaminação do canhão do cateter ou na ocorrência de infecção da corrente sanguínea relacionada ao cateter venoso central. Tal afirmação, entretanto, não tem força de evidência suficiente para recomendar o uso de um tipo específico de conector sem agulha, uma vez que esses melhores estudos compararam sistema aberto com sistema fechado, além de terem utilizado diferentes tipos de conectores.

infections. Ann Intern Med. 2000;132(5):391-402. Erratum in: Ann Intern Med. 2000;133(5):395. Comment in: ACP J Club. 2000;133(3):96.

10. Yoshida J, Ishimaru T, Fujimoto M, Hirata N, Matsubara N, Koyanagi N. Risk factors for central venous catheter-related bloodstream infection: a 1073-patient study. J Infect Chemother. 2008;14(6):399-403

11. United States Department of Labor. Occupational Safety \& Health Administration. FDA safety alert: Needlestick and other risks from hypodermic needles on secondary I.V. administration sets - piggyback and intermittent I.V. Rockville, 1992.

12. Zanetta DMT. Delineamento de estudos em medicina. In: Massad E, Menezes RX, Silveira PSP, Ortega NRS. Métodos quantitativos em medicina. Barueri: Manole; 2004. p. 389421.

13. Widmer AF. Related infections. In: Wenzel RP. Prevention and control of nosocomial infections. 2nd ed. Baltimore: Williams \& Wilkins; 1993. p 556-79.

14. Niël-Weise BS, Daha TJ, van den Broek PJ. Is there evidence for recommending needleless closed catheter access systems in guidelines? A systematic review of randomized controlled trials. J Hosp Infect. 2006;62(4):406-13. Comment in: J Hosp Infect. 2006;64(4):405-6.

15. Ramritu P, Halton K, Cook D, Whitby M, Graves N. Catheter-related bloodstream infections in intensive care units: a systematic review with meta-analysis. J Adv Nurs. 2008;62(1):3-21. Review. 